\title{
Characterization of an integrally wound tungsten and aluminum filament for physical vapor deposition
}

\author{
William Goble ${ }^{\mathrm{a} b}$ and Ricardo Ortiz \\ MMT Observatory $^{\mathrm{b}}$, University of Arizona, Tucson, AZ, USA 85721-0065
}

\begin{abstract}
As part of the effort to increase the reliability of the MMT Observatory (MMTO) 6.5m Primary Mirror Coating System, the specified filament has changed from a configuration in which the aluminum charge is hand wound around a tungsten filament to a configuration in which the aluminum is integrally wound with the tungsten at the time of filament manufacture. In the MMTO configuration, this filament consists of the three strands of tungsten wire and one strand of aluminum wire. In preparation of a full system test utilizing two hundred filaments fired simultaneously, an extensive testing program was undertaken to characterize these filaments using a four filament configuration in the MMTO small coating chamber $(0.5 \mathrm{~m})$ and then a forty filament configuration in the University of Arizona Steward Observatory coating chamber $(2 \mathrm{~m})$. The testing using the smaller coating chambers has shown these filaments provide very predicable coatings from test to test, and with the proper heating profile, these filaments greatly reduce the likelihood of aluminum drips. The initial filament design was modified during the course of testing by shortening the unwound filament length to closer match the aluminum load required in the MMTO coating chamber. This change increased the aluminum deposition rates without increasing the power delivered of the filament power supplies (commercial welders). Filament power levels measured at the vacuum chamber feedthroughs, currents, and deposition rates from multiple coating tests, including a full system test, are presented.
\end{abstract}

Keywords: physical vapor deposition, aluminization, tungsten filament, MMT Observatory

\section{INTRODUCTION}

During the 2010 in-situ coating of the MMTO 6.5m primary mirror, numerous molten aluminum droplets contacted the metallized polyester vacuum barrier that was separating the clean vacuum from the dirty vacuum. This resulted in a pressure increase near the optical surface of the primary mirror during the coating process, but more importantly, the damaged barrier emitted vapors which mixed with the aluminum being deposited on the mirror substrate. This combination of aluminum and polyester vapors left a distinctive brown haze in areas of the mirror coating. In order to prevent a similar incident during the next primary mirror recoating, the MMTO has embarked on a multiyear project to reduce the likelihood of aluminum droplets falling from the filaments and contacting the vacuum barrier. This is to be accomplished through three major improvements: converting control of the filament heating process from a manual operation to computer control ${ }^{[1]}$, protecting the vacuum barrier with a shield, and changing the filaments used in the process to an integrally wound aluminum and tungsten filament. The integrally wound style filament was selected based on the filament used in the Carnegie Observatories Magellan 6.5m coating chamber (Frank Perez, personnel communication, 2012), and then modified and characterized to work in the MMTO 6.5m coating chamber.

The MMTO $6.5 \mathrm{~m}$ coating chamber is a filament based physical vapor deposition system. The filaments are arranged in three concentric rings with the filaments electrically connected in ten arrays of twenty filaments each. All 200 filaments are heated simultaneously by applying voltage across to the filaments using voltage controlled direct current sources (commercially available welders) ${ }^{[2]}$.

\footnotetext{
a Contact email: wgoble@mmto.org

${ }^{b}$ The MMT Observatory is a joint facility of the Smithsonian Institution and the University of Arizona.
} 


\section{NEW VERSE OLD FILAMENTS}

For the last two MMTO primary mirror coatings (2005 and 2010), the filaments were made from three strands of $0.51 \mathrm{~mm}(0.020 \mathrm{inch})$ diameter tungsten wire. After the tungsten filaments were delivered from the vender, MMTO personnel would manually wrap aluminum coils around the tungsten, figure 1 . The total amount of aluminum loaded in this manner was 0.6 grams. During the filament heating process, the aluminum coils would melt and leave multiple balls of aluminum dangling from the coils. As the filaments continued to heat up during the process, the aluminum balls would be wicked up into the filament and evaporated. Figure 2 shows a filament after a single coating in which two of these aluminum balls remained on the filament at the end of the coating and have solidified.

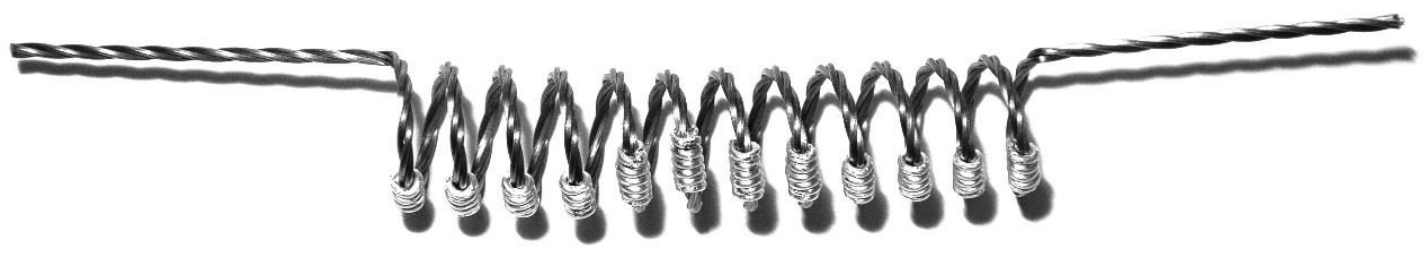

Figure 1 - 2010 MMTO tungsten filament loaded with $0.6 \mathrm{~g}$ of aluminum

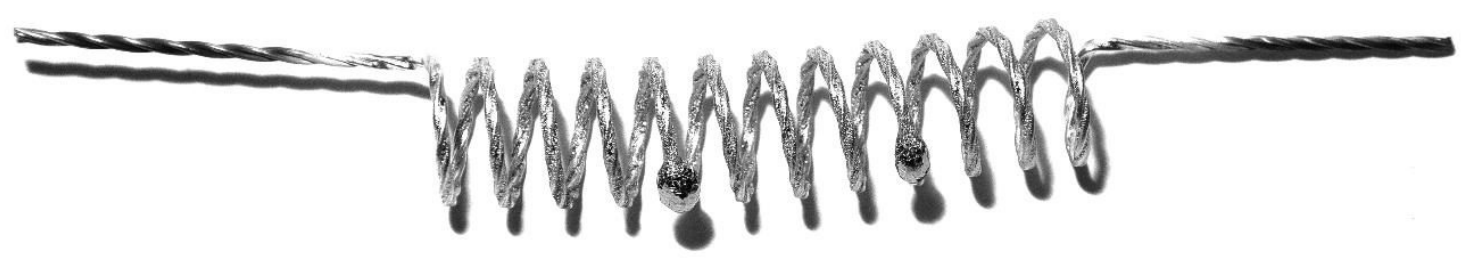

Figure 2 - 2010 MMTO tungsten filament after a single coating

Since the process of manually loading these filaments is labor intensive and is perceived as being inconsistent from filament to filament, an integrally wound aluminum and tungsten filament was selected for further evaluation. This style of filament had been successfully utilized in the Magellan $6.5 \mathrm{~m}$ Coating Chamber, and the same vender, Midwest Tungsten Service, Inc. ${ }^{c}$, was contacted to fabricated a slightly different filament to match the MMTO specifications, particularly a larger aluminum charge and a different filament geometry. This filament is made from three strands of 0.64 $\mathrm{mm}(0.025 \mathrm{inch})$ diameter tungsten wire wound around $0.64 \mathrm{~mm}(0.025 \mathrm{inch})$ aluminum wire core. To change the amount of aluminum available for evaporation, the number and size of the coils in the center section of the filament are adjusted. Inherently, more tungsten must to be added to the filament in order to increase the aluminum charge.

The first extensively tested filament was designated 23H by Midwest Tungsten Service Inc., figure 3. Initially, the desire to keep the cold filament resistance similar between the old and new style filaments drove the filament configuration. Since with the new filaments the aluminum wire is an electrical conductor when the filament is cold, the $23 \mathrm{H}$ contained much more wire in the coil section than necessary. The cold electrical resistance of the $23 \mathrm{H}$ filament is approximately $0.036 \Omega$ compared to the $0.045 \Omega$ of the old filament. Similarly due to the amount of wire in the coil section, the amount

\footnotetext{
${ }^{c}$ Midwest Tungsten Service Inc., Willowbrook IL, USA, www.tungsten.com
} 
of aluminum in the coil section of the filament was approximately 0.85 grams. This is over four times the 0.20 grams required in the MMTO coating chamber for a $1000 \AA$ primary mirror coating ${ }^{[3]}$.

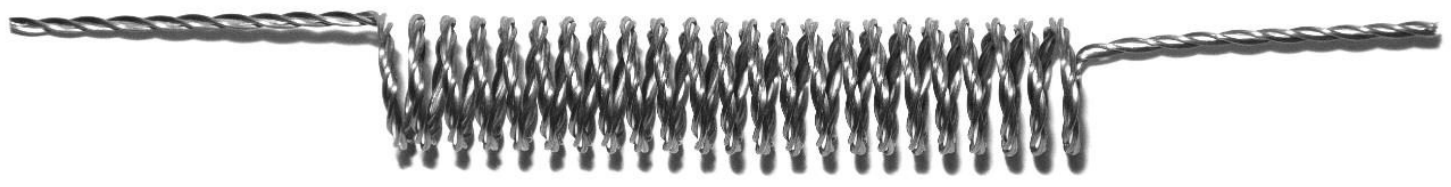

Figure 3 - MMTO specified filament, designated 23 H by Midwest Tungsten

\section{INITIAL SUNNYSIDE TESTING}

Since significant changes had been made to the MMTO coating system after the 2010 coating, a testing campaign utilizing a fifth scale system was performed at the University of Arizona Steward Observatory's Sunnyside coating facility. With minor modifications, the Sunnyside $2 \mathrm{~m}$ coating chamber was reconfigured to test 40 filaments using two arrays of 20 filaments each. Both arrays were heated simultaneously using the MMTO DC power supplies controlled by the MMTO developed software. Additionally a $0.5 \mathrm{~m}$ coating chamber was utilized to test two arrays consisting of only two filaments each. The primary function of the $0.5 \mathrm{~m}$ chamber was to verify the effect of any changes to the process control software.

In conjunction with the control system development, a number of coating tests using the $23 \mathrm{H}$ filament were performed. Only a few drips were observed from these tests, but the deposition rates observed when using the $23 \mathrm{H}$ filaments in the Sunnyside $2 \mathrm{~m}$ chamber did not meet the MMTO goal of $50 \AA / \mathrm{s}$. While the maximum power output of the DC power supplies was not being utilized during the two array tests, when scaled to full size, the new system and filaments would require much more incoming $\mathrm{AC}$ power than available for the coating system at the telescope. To improve the deposition rate without increasing the power from the existing DC supplies, the filament geometry was manipulated to reduce to the amount of tungsten and aluminum in the coil section of the filament.

\section{ALUMINUM AVAILABLE FOR EVAPORATION FROM AN INTEGRAL WOUND FILAMENT}

A number of filaments were reworked by hand to contain different amounts of aluminum in the coil section of the filament. A series of quick tests were performed in the $0.5 \mathrm{~m}$ chamber to determine the percentage of aluminum that would be evaporated if sufficient power were applied to the filaments. During all of these tests, power was applied to all of the filaments until the recorded deposition rate dropped to zero. Conservatively, at least $73 \%$ of the aluminum in the coil section of all filament configurations was evaporated, figure 4 . The variation in the data may possibly be due to the imprecise method used to fabricate these test filaments. However for the purpose of specifying the length of tungsten and aluminum wire in the coil section of the filament, a conservative approach to determine the amount aluminum charge was to assume $73 \%$ of the total aluminum in the coil section of the filament would be available to be evaporated. Since the aluminum in the mounting legs of the filament will never melt, the aluminum contained in this section of the filament was not included in this calculation. 


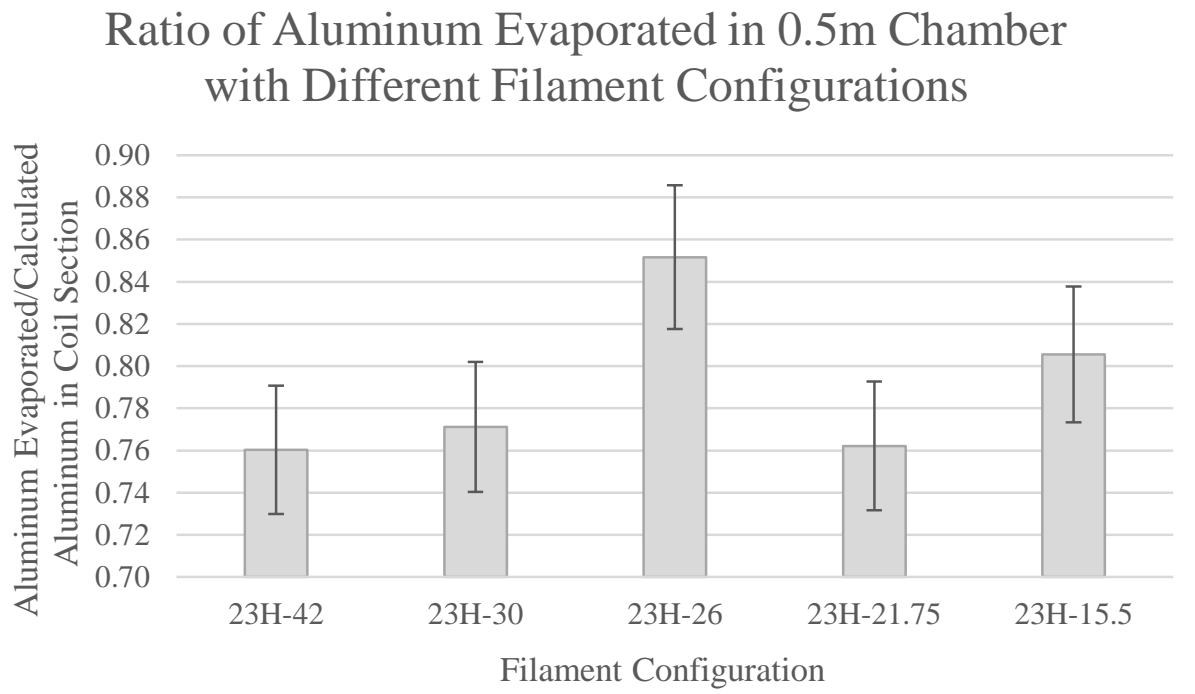

Figure 4 - Ratio of aluminum evaporated to calculated aluminum in the coil section of the filament. The tested filaments were hand fabricated from $23 \mathrm{H}$ filaments. The last two numbers of the filament configuration is the overall unwound filament length in inches.

\section{SUNNYSIDE TESTING WITH RECONFIGURED FILAMENT}

Based on the available aluminum experiments, a filament configuration with a mass of just under 0.4 grams aluminum in the coil section was selected. This filament has 13 wraps in the coil section and a manufacturer designation of $13 \mathrm{H}$, fig 5 . Once selected, the $13 \mathrm{H}$ filament has been utilized for all subsequent coating system test. The as-received overall mass of these filaments is on average 12.32 grams which is nominally 8.1 grams less the $23 \mathrm{H}$ used in the early stages of control system testing.

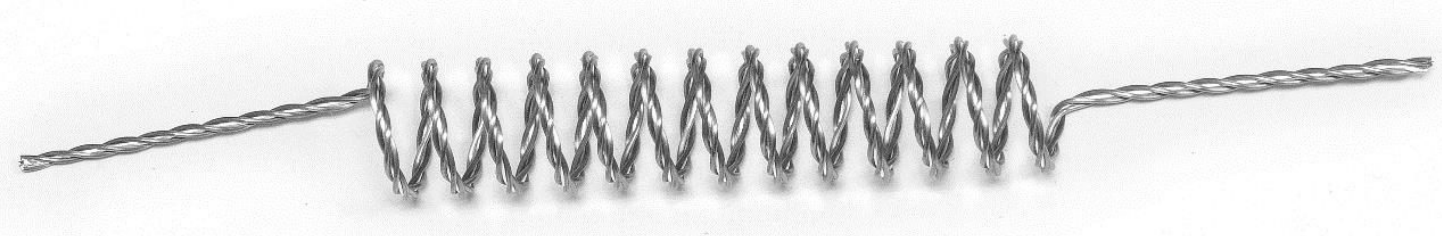

Figure 5 - Refined MMTO specified filament designated 13 H by Midwest Tungsten

\subsection{Reduction in mass and the effect on deposition rate}

In the evolution of the testing at Sunnyside, both the $23 \mathrm{H}$ and $13 \mathrm{H}$ were tested in the converted Sunnyside $2 \mathrm{~m}$ coating chamber using the same filament heating profile. For a fixed maximum power level for each array, in this case $9600 \mathrm{~W}$, the $13 \mathrm{H}$ has a higher deposition rate, figure 6 . Although the $13 \mathrm{H}$ filament has almost twice the aluminum necessary for a $1000 \AA$ coating in the MMTO $6.5 \mathrm{~m}$ coating chamber, the filaments are intended to be replaced after each coating. This is primarily due to limited amount of testing performed on pre-fired filaments. If resources permit in the future, the amount of aluminum in the coil section of the $13 \mathrm{H}$ filament may be reduced again in order to achieve an additional increase the deposition rate. For all of the data presented, the power at load was calculated from the voltage measured at the vacuum chamber feedthroughs and the current measured at the DC power supplies. The calculated power at load was then averaged between the two arrays. Similarly, the deposition rate was averaged between two crystal monitors that were located within $25 \mathrm{~cm}$ (10 inches) of each other. 

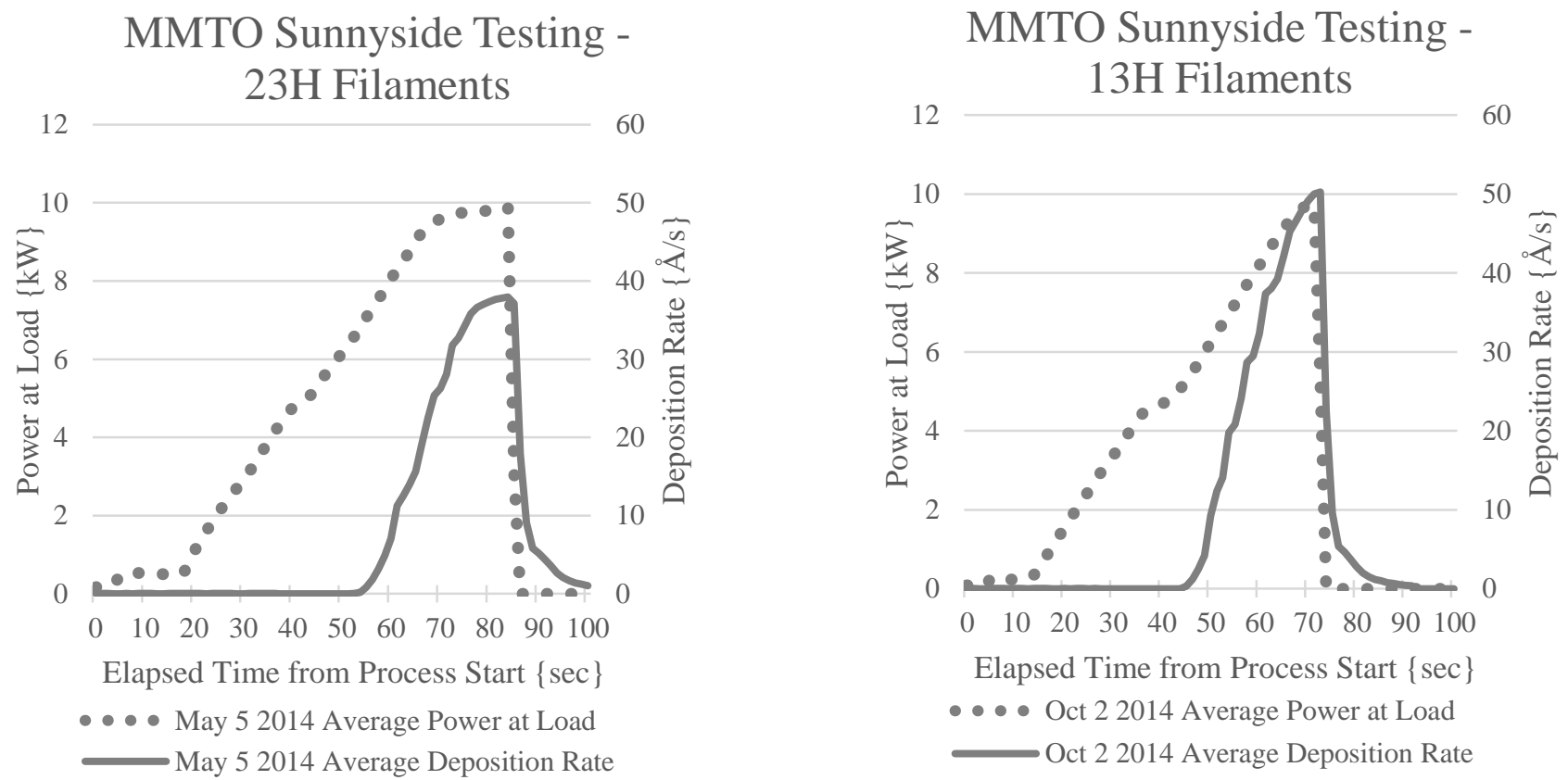

Figure 6 - Deposition rate comparison between $23 \mathrm{H}$ and $13 \mathrm{H}$ filaments. The power profile in both test cases is almost identical, but due to the increased rate, the Oct 2014 achieve the required coating thickness earlier.

\subsection{Aluminum drips during Sunnyside testing}

For a number of tests in the Sunnyside $2 \mathrm{~m}$ coating chamber, stainless steel trays were installed directly under the filament arrays to provide a visual indication of aluminum drips that occurred during the coating process. In the early stages of testing the $13 \mathrm{H}$ filaments, one or two drips from the 40 filaments would be evident after each test. This was attributed to a filament heating curve that allowed the molten aluminum to remain on the filament longer than necessary.

Since the implementation of the computer control filament heating curve, there was always a power hold step at 4800 watts. However for most of the control system testing, this power hold was never really achieved due to the manner in which the transition criteria from this process state was defined. In figure 6 above, power pauses at the level of 4800 watts for only a very short duration. As the control system testing became more mature, this mid stage power hold became more pronounced. Based on the changes in the current during the process, the 4800 watts may have been at a point in the process in which the aluminum on the filament has already melted. This mid process power hold may not be necessary at all, but due to the manner in which the original control system software was written, the mid process power hold could not be bypassed without major software changes. Hence, a new power hold target of 2200 watts was selected; at this point in the process, the filaments should not yet be at a high enough temperature for the aluminum to have melted. This was intended to reduce the time molten aluminum is present on the filament. No drips were observed during the two subsequent $2 \mathrm{~m}$ Sunnyside coating chamber tests which took place on September $9^{\text {th }}$ and $18^{\text {th }}, 2015$. The power input to the filaments was identical for these two tests, and subsequently, the deposition rates for both tests were almost identical, figure 7.

Interestingly when the power level for the hold step was reduced, two distinct peak and valley features were observed in the electrical current. This area is circled in figure 8 . These local changes in current could be attributed to phase changes in the aluminum which would affect filament resistance and hence the current drawn by the filaments. Using images from a filament viewing camera, the second valley corresponds reasonably well with point in the process where the molten aluminum droplet is being wicked back into the filament. Unfortunately due to automatic camera gain adjustments, an image of a filament was not captured when the process moved through the first valley. No drips were observed during a post coating inspection of the drip shields for either test. 
September 9th, 2015

MMTO Sunnyside - $13 \mathrm{H}$ Filaments

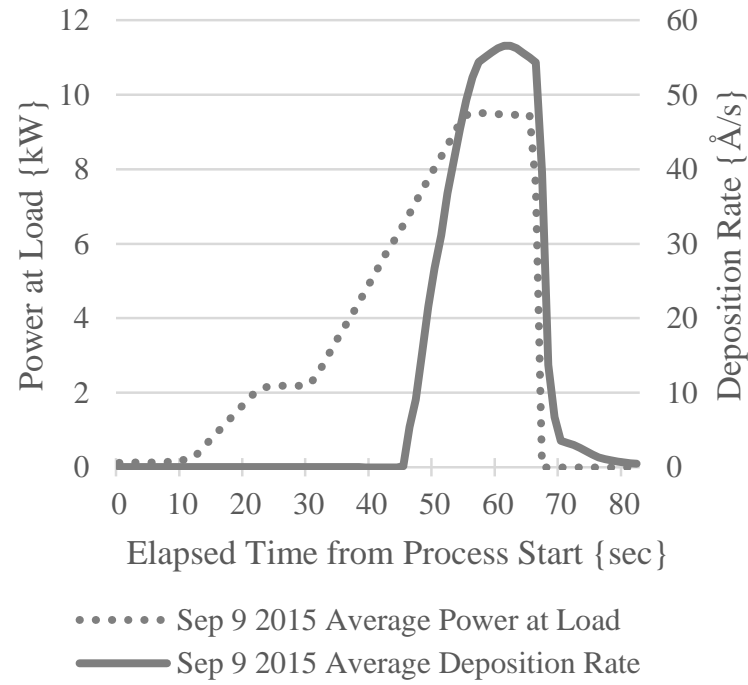

September 18th, 2015

MMTO Sunnyside - $13 \mathrm{H}$

Filaments

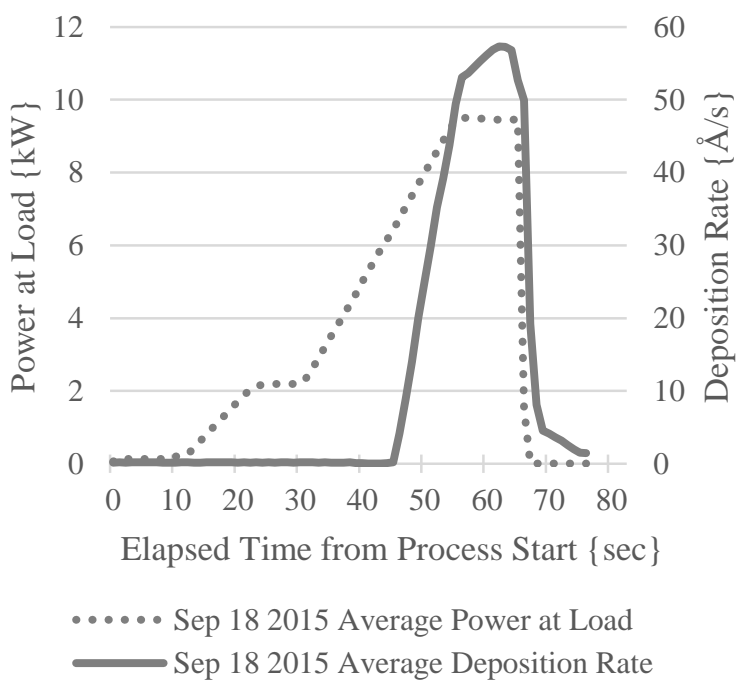

Figure 7 -Sept. 9th, 2015 and Sept. 18th, 2015 MMTO two filament array testing at Sunnsyide. The same process parameters were used for both tests.

\section{September 18th, 2015 MMTO Sunnyside Testing - 13H Filaments}

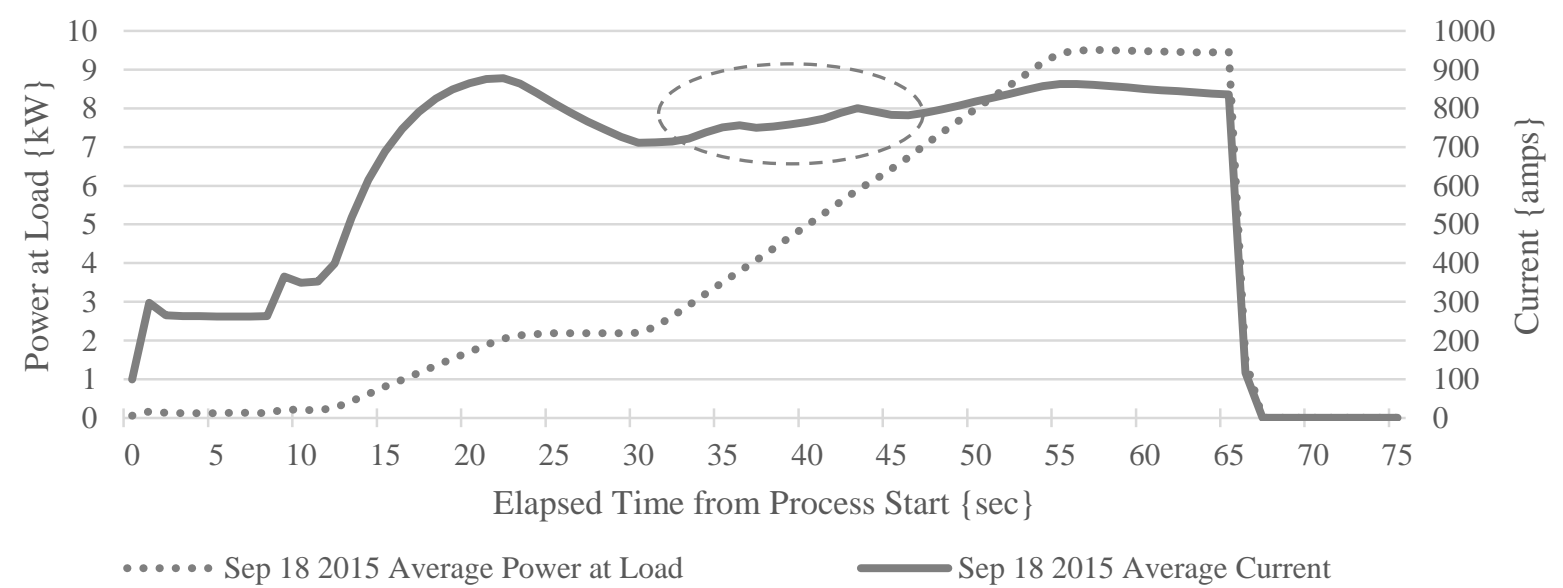

Figure 8-Sept. 18th, 2015 MMTO two filament array testing at Sunnyside. The local changes in the current drawn by the filaments while the power is increasing could be attributed to resistance changes in the filament due to the phase change of the aluminum.

\section{FULL SCALE SYSTEM TESTING}

As a parallel effort to the filament and control system development, a complete MMTO coating system capable of performing full system testing was assembled at the Fred Lawrence Whipple Observatoryd Base Camp facility in Amado, AZ. Utilizing much of the support equipment necessary for an in-situ coating at the telescope, this test setup

\footnotetext{
${ }^{d}$ Fred Lawrence Whipple Observatory is operated by the Smithsonian Institution.
} 
uses the vacuum head (Bell Jar) containing ten arrays of 20 filaments each and includes a witness slide holder located at approximately the same position as the primary mirror surface relative to the filament arrays.

In between the last round of Sunnyside testing and the full system tests at Base Camp, the filament power control system was converted from active control through a software state machine to a simpler open loop playback mode. The playback mode increases the DC voltage applied to the filaments according to a predefined filament heating curve. For the two full system tests, the filament heating curve followed the same power profile utilized during the September $9^{\text {th }}$ and $18^{\text {th }}, 2015$ MMTO tests in the Sunnyside $2 \mathrm{~m}$ chamber. The full scale tests at Base Camp were completed on May $4^{\text {th }}, 2016$ and May $11^{\text {th }}$, 2016. However, only data from the May $4^{\text {th }}$ system test has been presented, for the results from the May $11^{\text {th }}$ test almost perfectly overlay the data from the earlier test. Both tests utilized $13 \mathrm{H}$ filaments and the open loop control software.

\subsection{Power at load, currents and deposition rates from full scale testing}

Due to differences in filament geometry and bus bar resistance, the results from the full scale tests have slightly different characteristics than the tests at Sunnyside, figures 9 and 10. The May $4^{\text {th }}, 2016$ full scale test recorded a $1000 \AA$ thick coating at the two deposition monitor crystals located in the chamber at a point corresponding to primary mirror vertex. However, the maximum deposition rate of $32 \AA /$ s (averaged between the two center deposition monitors) is below the MMTO goal of $50 \AA$ /s. Fortunately, the average array current seen in the MMTO Bell Jar is lower than the average current observed at Sunnyside; since the DC power supplies have a current limit of approximately 990 amps, the power applied to the filaments could potentially be increased in order to increase the deposition rate. Additionally, the local changes in current are at a different point in the process than experienced at Sunnyside, and the need for the mid process power hold (2000 watts during the full system test) may be unnecessary. With the simplified open loop software control, the power at load tracks a very similar profile to the one used at Sunnyside. A significant exception would be the maximum power at load for a single filament array during the full scale test was just below 9000 watts; this is compared to the 9600 watts experienced at Sunnyside. While the software simplification was stable and performed well, minor tweaks to the power profile could potentially increase system performance. If resources permit, one more full system test will be performed before the summer 2016 primary mirror coating in order to slightly adjust the filament heating profile.

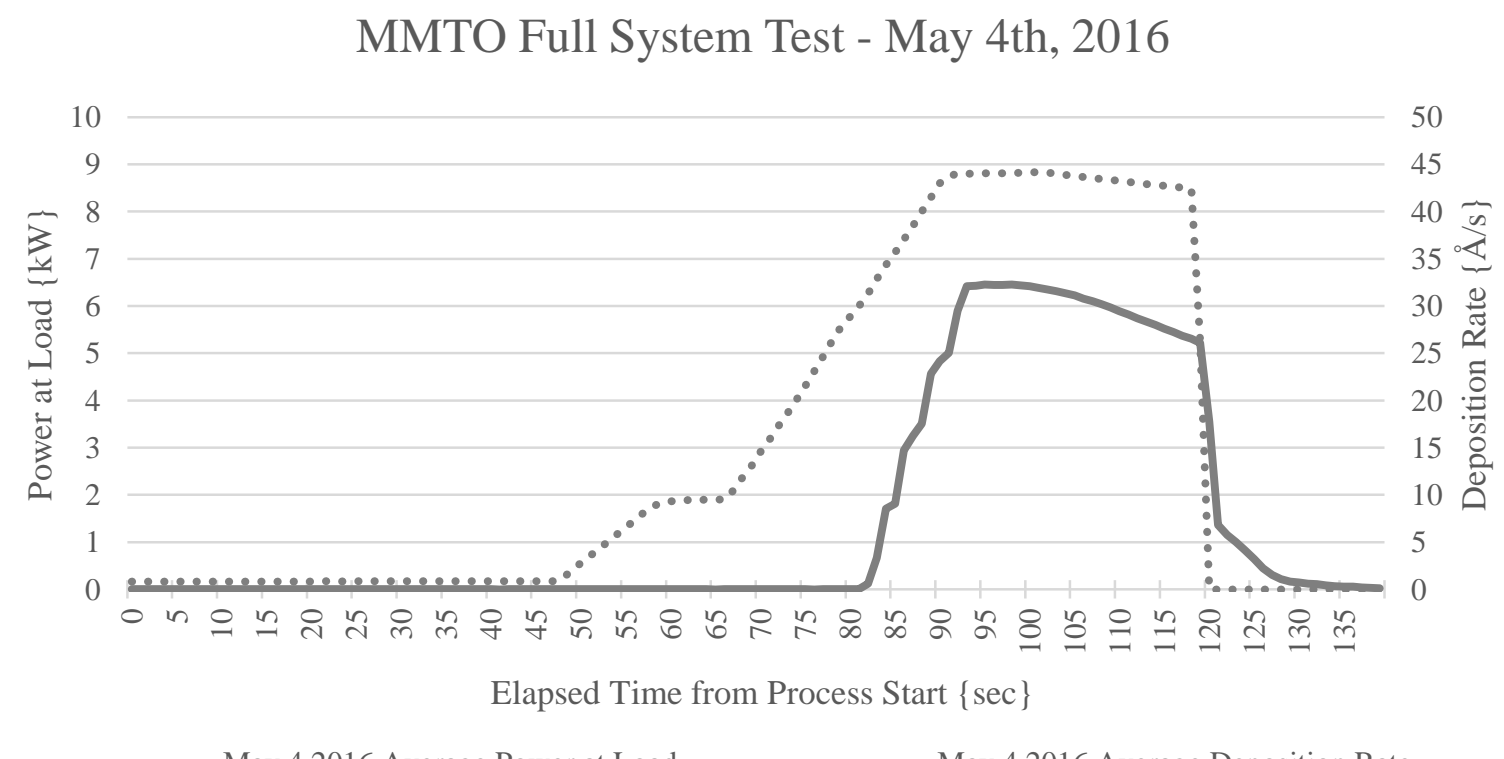

Figure 9 -Power at load and deposition rate from the May $4^{\text {th }}, 2016$ full scale coating system test. 


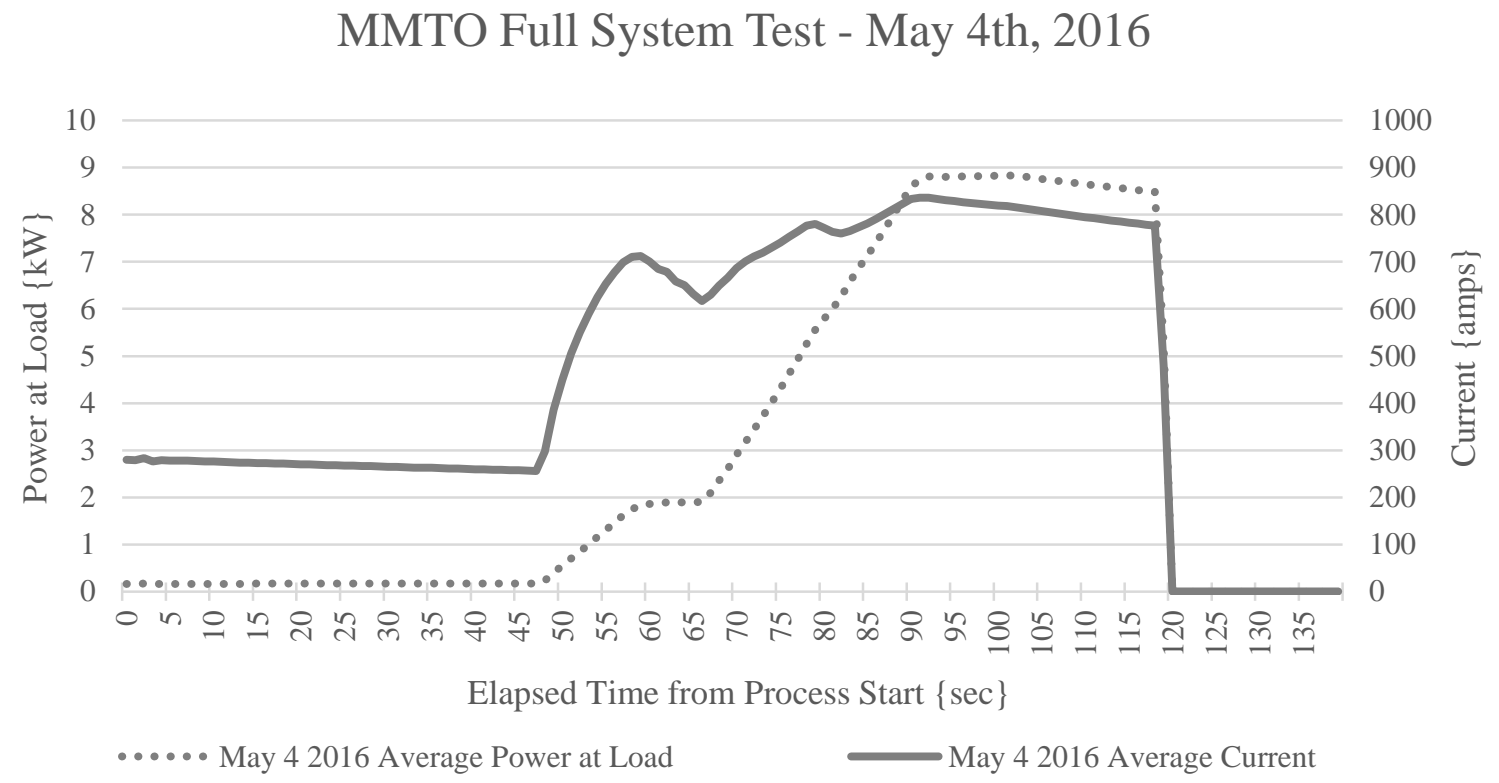

Figure 10 - Load power and current from the May $4^{\text {th }}, 2016$ full scale coating system test.

\subsection{Drips observed during full scale system testing}

During the May $11^{\text {th }}, 2016$ full scale test, a sheet of metallized polyester was placed in the coating chamber at the approximate location of the vacuum barrier used at the telescope during a primary mirror recoating. Inspection of the metallized polyester after the test revealed 11 small discolorations from molten aluminum contacting the sheet during the coating. All of the discolorations are less than $0.75 \mathrm{~mm}$ (.030 inches) in diameter, and the integrity of the metallized polyester does not appear to be compromised at any of the discolored areas.

\section{CONCLUSION}

The MMTO has been fortunate enough to be able to invest a large amount of effort to gain some experience with the integrally wound tungsten and aluminum filaments before actually performing a primary mirror coating. When used for a single coating, these filaments have provided very consistent performance. Through the evolution of the coating system tests, the $13 \mathrm{H}$ filament has been selected for use with the next MMTO primary mirror coating which is scheduled for the summer of 2016. Although the amount of tungsten and aluminum in the coil section of the $13 \mathrm{H}$ filament could probably be reduced, large performance gains are not likely to be achieved from this change. With the proper input power profile, the integral wound tungsten and aluminum filament is capable of greatly reducing the likelihood of amount of molten aluminum dripping from the filament during a coating.

\section{REFERENCES}

[1] Gibson, J.D., D. Clark, D.Porter, "Software framework for the 2014 MMT Observatory primary mirror realuminization", Proc. SPIE 9152, Software and Cyberinfrastructure for Astronomy III, 91521K (2014)

[2] Clark, D., W. Kindred, J.T. Williams, "In situ aluminization of the MMT 6.5m primary mirror", Proc. SPIE 6273, Optomechanical Technologies for Astronomy, 627305 (2006)

[3] Kindred, W., J.T. Williams, and D. Clark, "In-Situ Aluminization of the MMT 6.5m Primary Mirror", MMTO Technical Report \#03-8 (2003) 